\title{
A SESSION-BASED COLLABORATION ENVIRONMENT FOR BIM-BASED PROJECT LIFE-CYCLE MANAGEMENT
}

\author{
Sangyoon Chin \\ Dept. of Civil, Architectural, \\ and Environmental System Engineering \\ Sungkyunkwan Univ. Suwon 400-746, S. Korea \\ schin@skku.ac.kr \\ Tae-Hong Shin \\ Dept. of Civil, Architectural, \\ and Environmental System Engineering \\ Sungkyunkwan Univ. Suwon 400-746, S. Korea \\ cmcic@skku.edu

\section{Cheolho Choi} \\ Doalltech \\ Sae-myung B/D 770-11, Yeoksam-Dong, \\ Gangnam-Gu, Seoul, Korea 135-928 \\ choi@doalltech.com
}

\author{
Su-Won Yoon \\ Doalltech \\ Sae-myung B/D 770-11, Yeoksam-Dong, Gang- \\ nam-Gu, Seoul, Korea 135-928 \\ yoonsuwon@skku.edu \\ Ghang Lee \\ Dept. of Architectural Engineering \\ Yonsei Univ. Seoul 120-749, \\ S. Korea \\ glee@yonsei.ac.kr

\section{Soon-Wook Kwon} \\ Dept. of Civil, Architectural, \\ and Environmental System Engineering \\ Sungkyunkwan Univ. Suwon 400-746, S. Korea \\ swkwon@skku.edu
}

\begin{abstract}
It has been recognized that a system that facilitates efficient communication and interaction is critical in order to improve coordination between AEC project participants. Various research literatures show that migration of conventional AEC work processes, which have relied heavily on two-dimensional drawings, to more convergent processes that share a single common information source based on three-dimensional object-oriented data or BIM, can yield better outcomes and improve productivity. However, several problems made it very difficult to collaborate and share information between project participants. Some of the problems include legal liability issues, a reluctance to share inside information with others, maintenance of a master model, a dynamically changing organizational structure of a project group, and a collaboration-support system that can support these issues, etc. Therefore, the objective of this paper is to propose a conceptual scheme of a "session-based" collaboration environment, called Construction Project Life Cycle Management (CPLM). CPLM is a system intended to help project participants make better decisions and to facilitate coordination between various stakeholders in a project by providing virtual collaboration groups called "sessions" and methodology to support the open information policy between both horizontal and vertical organizations within those sessions. Additionally, this paper introduces a system to be developed using the proposed conceptual scheme of a session-based CPLM and its development plan.
\end{abstract}

\section{KEYWORDS}

BIM, Session-based CPLM(Construction Project Life-cycle Management), Collaboration 


\section{INTRODUCTION}

Fragmentation of the Architecture, Engineering, and Construction (AEC) industry has resulted in poor coordination between project participants. Such ill-coordinated operations cause waste in the work process, which include frequent reworks and redundant operations. It has been recognized that a system that facilitates efficient communication and interaction is critical in order to improve coordination between AEC project participants.

Various research literatures show that migration of conventional AEC work processes, which have relied heavily on two-dimensional drawings, to more convergent ones that share a single common information source based on three-dimensional objectoriented data, can yield better outcomes as well as improved productivity. Building Information Modeling (BIM) is the result of these efforts [1-3]. However, the application of the new model-driven processes in real projects revealed several problems: (1) the industry is more process-driven rather than its final product; (2) each AEC project features vastly different artefacts than other projects; (3) collaboration between the participants doesn't last beyond project completion; (4) collecting semantically consistent information sets (i.e. BIM data) throughout the project's life span is difficult due to frequent change orders.

In the beginning of the BIM adoption process, they realized that collaboration processes lay at the core of BIM rather than the building information models. However, several problems made it very difficult to collaborate and share information between project participants. Some of the problems include legal liability issues, a reluctance to share inside information with others, maintenance of a master model, a dynamically changing organizational structure of a project group, and a collaboration-support system that can support these issues, etc.

This paper proposes a conceptual scheme for a "session-based" collaboration environment, called Construction Project Life Cycle Management (CPLM). CPLM is a system intended to help project participants make better decisions and to facilitate coordination between various stakeholders in a project by providing a mechanism to create virtual collaboration groups called "sessions" and a methodology to support information exchange between both horizontal and vertical organizations within those sessions. It tries to tackles the issues of who maintains the master building information model throughout the project's life-cycle and of how to propagate the changes without causing legal issues. Additionally, this paper introduces a system to be developed using the proposed conceptual scheme of a session-based CPLM

\section{BIM AND COLLABORATION IN CONSTRUCTION INDUSTRY}

BIM (Building Information Modeling) has been variously defined in various ways by researchers in the AEC industry [1-3]. Such definitions of BIM include various concepts including object-oriented 3 dimensional data, processes, information, interoperability, reuse, a building life-cycle, among others. BIM aims to improve productivity through reuse of information, collaboration between project participants, and the early involvement of engineers in projects.

Recently, BIM has been applied to more construction projects in order to improve productivity and quality. However, most of these projects applied the system only in part for example, design or construction simulation due to the absence of BIM-based collaboration environments that provided proper method, process, and interoperability through the construction project life-cycle.

There have been various existing efforts and studies to support collaboration environments for the AEC industry [4]. However, many of them focused on the support of traditional work processes rather than collaboration through BIM.

On the other hand, the manufacturing industry has been operated based on a collaboration environment called PLM (Product Life-cycle Management). Although there are significant differences between the AEC industry and the manufacturing industry, we proposed a framework for CPLM (Construction Project Life-cycle Management) by benchmarking PLM in the manufacturing industry and reflecting the characteristics of the AEC industry. 


\section{CHARACTERISTICS OF CONSTRUCTION PROJECT LIFE-CYCLE}

The life-cycle of a construction project consists of various phases from the requirement definition phase to the operation and maintenance phase as shown in Figure 1. Throughout the entire lifecycle of a building, more than hundreds of teams are involved and numerous building information models are generated according to the intents of participants.

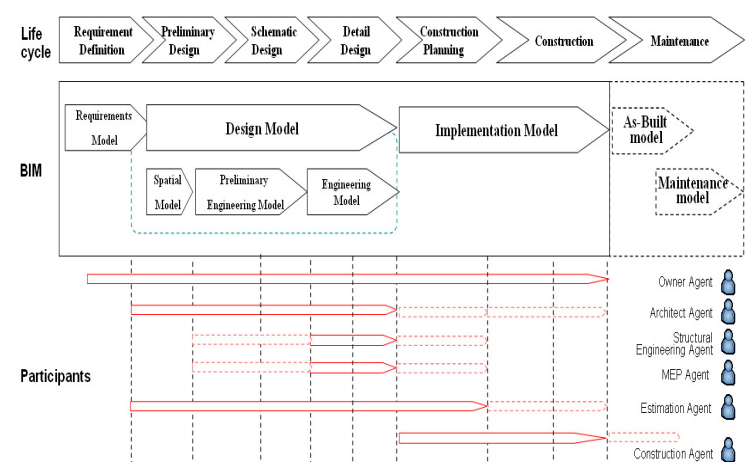

Figure 1. Participants and Building Information Models Involved in the Traditional Process of Building Construction

The processes of construction projects may vary depending on contract types. Figure 2 depicts the organization break-down structure (OBS) of the design-bid-build method that is commonly used in the AEC industry. As shown in Figure 2, the organizational structure of a construction project is hierarchical. This structure makes project participants difficult to share data and to collaborate. Thus, the traditional design-bid-build method does not fit BIM-based construction processes.

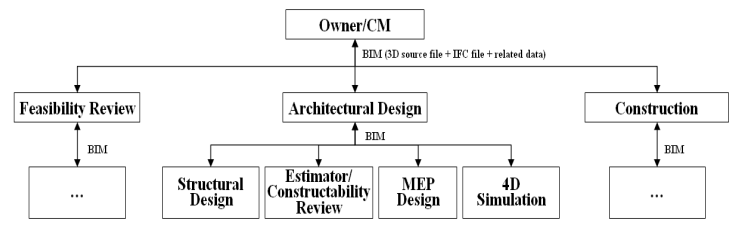

Figure 2. An Organization Breakdown Structure of the Design-Bid-Build Method

\section{THE CONCEPT OF CPLM AND A SESSION-BASED CPLM SYSTEM}

The main idea of CPLM is to support various contractual arrangements, which can be quite different and unique depending on construction projects, to accommodate the share and exchange of BIM among project participants throughout the life-cycle. To do so, a session-based collaboration framework has been developed in this research.

The meaning of session is defined as "a meeting or series of meetings of a body (as a court or legislature) for the transaction of business [5], and this research introduces two kinds of sessions to support a collaboration environment; one is a "contractual session" that lasts until the contract is expires, and the other is a "working session" that last temporarily during a certain period of time. With these two kinds of sessions, a collaboration mechanism for CPLM was developed using both kinds of sessions to support the share, exchange, and change management of BIM consistently throughout the project life-cycle.

The system that supports session-based CPLM is called a session-based CPLM system. Depending on the user types, functions of the session-based CPLM system can be categorized into two; one is the session-master function and the other is the session-user function. Session managers can create and manage sessions, user accounts, and access rights, and have access to any building information models in any sessions that they are in charge of. Users have access only to sessions that are invited to. However, users can create their own sessions and become a new session master. The relations between session masters and session users are often contractual. Session users can access their own building information models, but may or may not be able to access others depending on the contractual conditions. Those contractual relations and different information-access rights can be defined in the session-based CPLM system.

This research is a part of a virtual construction system development that was established with the support of Korea Ministry of Construction and Transportation, and KICTEP (Korea Institute of Construction and Transportation Technology Evaluation and Planning). Two system develop- 
ments have been scheduled; one is a prototype system to implement the proposed concept, and the other is a commercialized system to be practiced in real construction projects. Currently, a prototype system is being developed based on the proposed "session based collaboration environment".

This section will describe the system's architecture and information model, as well as future development plans.

\section{CPLM ARCHITECTURE}

Based on the proposed "session-based collaboration environment", CPLM system has been developed to support effective BIM-based building life-cycle management as well as collaboration among various project participants including the owner, architects, structural engineers, MEP engineers, estimators, and general contractors, etc. The system's architecture of a session-based CPLM is shown in Figure 3.

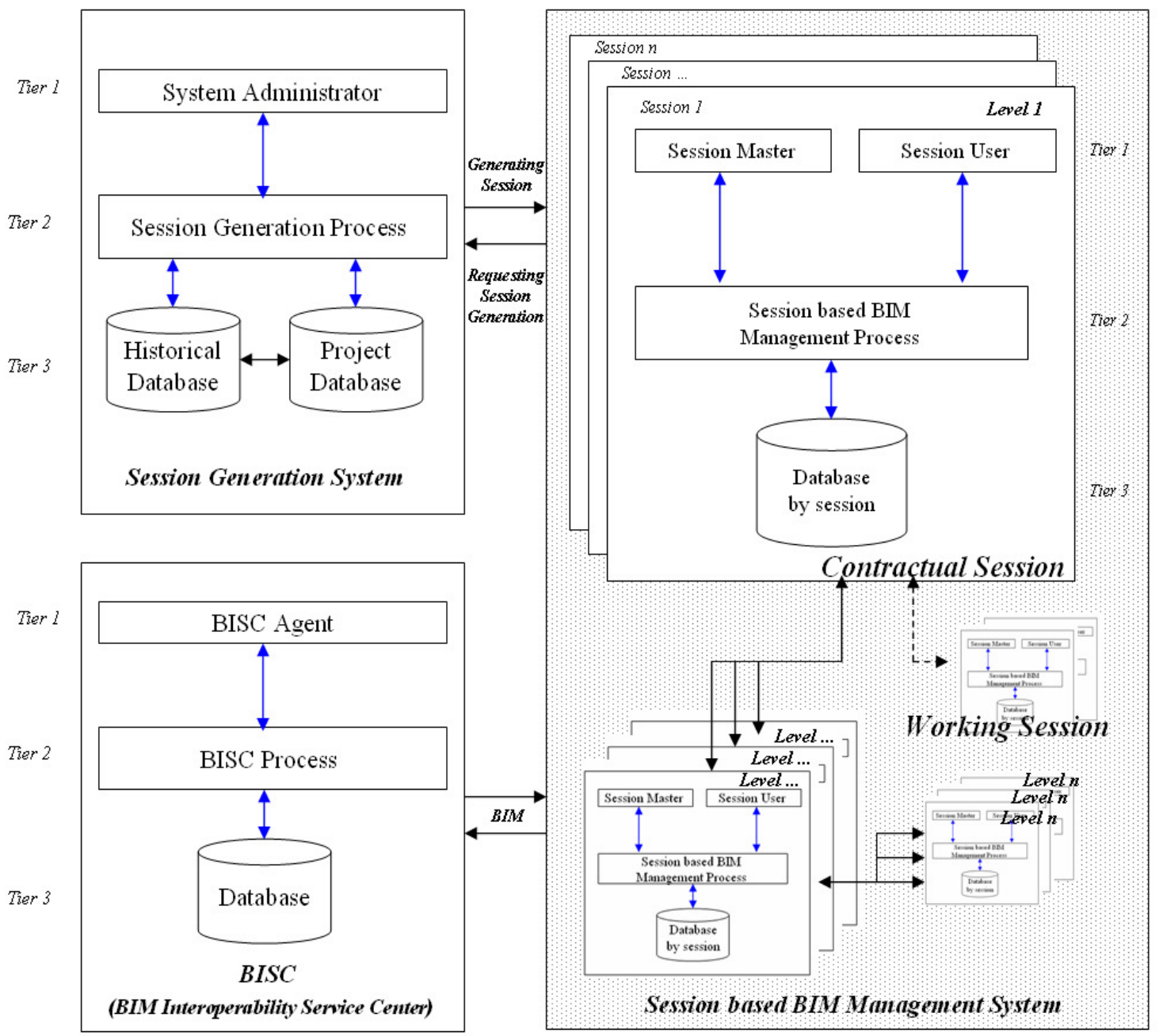

Figure 3. The Architecture of a Session-based CPLM System 


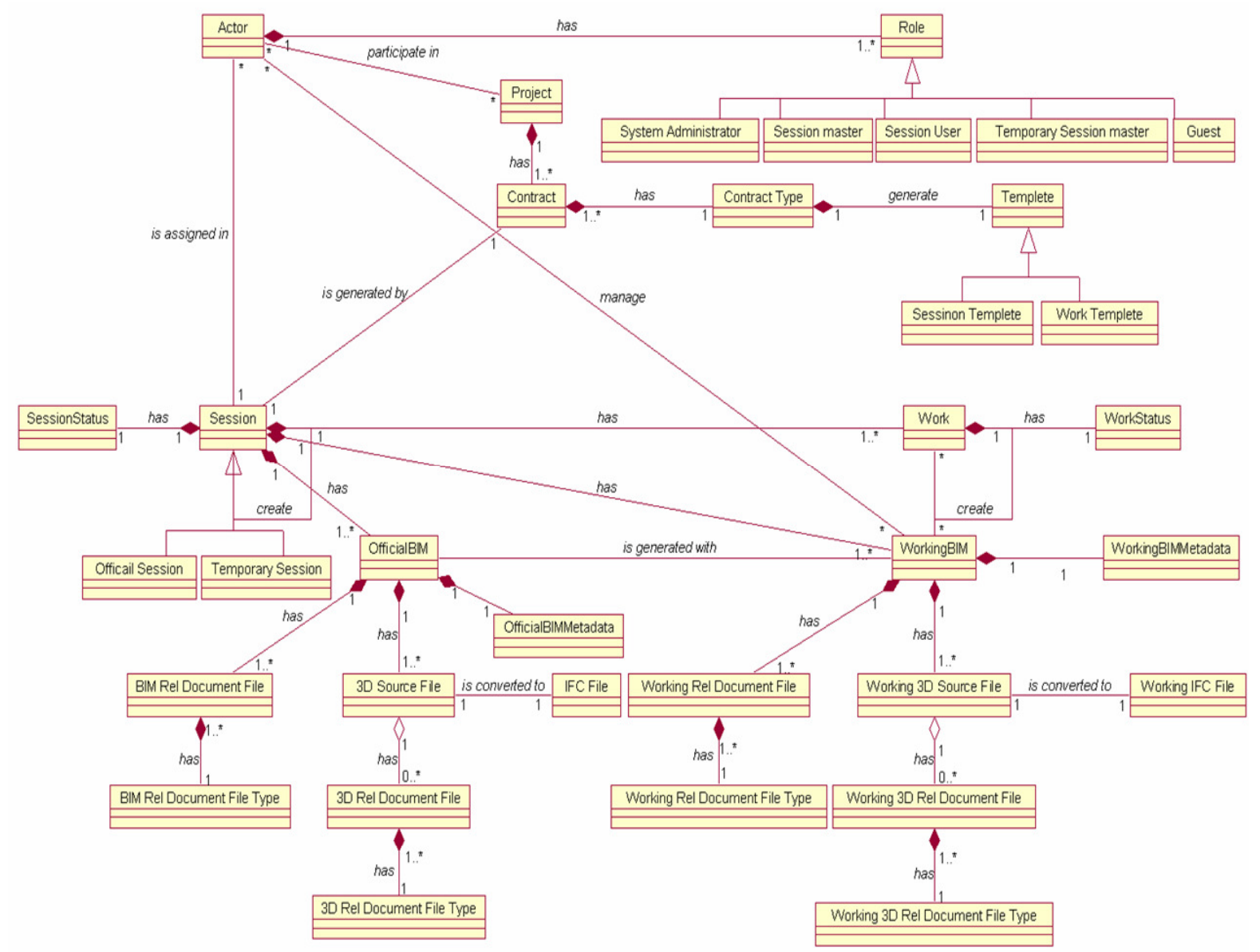

Figure 4. The Information Model of the Session-based BIM Management System

The CPLM system consists of three components: 1) the Session Generation System that allows designated users to generate sessions, 2) the Session-based BIM Management System that allows project participants to access and manage a building information model, and 3) the BIM Interoperability Service Center (BISC) that provides services related to technical interoperability issues.

The Session-based BIM Management System is the core of CPLM. It has a hierarchy structure.

\section{THE INFORMATION MODEL OF THE SES- SION BASED BIM MANAGEMENT SYSTEM}

The information model of the Session based BIM Management System, the core module of the CPLM system is described using the class diagrams of the UML (Unified Modeling Language). Figure 4 shows the class diagrams of Session based BIM Management System. The information model of the Session based BIM Management System consists of various classes; Project, Actor, Role, Contract, Session, Work, Approved BIM, Working BIM, etc., and the relationships between them. 


\section{CONCLUSION}

In order to improve the communication between participants in the fragmented design, engineering, and construction processes, this paper proposes a concept of session-based CPLM and introduces its framework.

The CPLM system has been proposed to facilitate coordination and communication among various stakeholders in a project by providing a mechanism to create "sessions". Sessions are virtual collaboration groups, which allow project participants to share information. There are session masters, who create and manage sessions, and session users, who are invited to participate in sessions. The information access rights are often defined based on contractual agreements. A session can be easily opened and closed by a session master.

However, a session can be kept permanently. In any case, the history data will be archived. The permanent session is a "contractual session" that is generated by contracts. The other one, which is temporarily generated to support collaboration among project participants, is called a "working session".

Presently, our research team is developing the prototype system of the session-based CPLM System" that is composed of three units: i.e., the Session Generation System, the Session-based BIM Management System, and the BIM Interoperability Service Center (BISC). This paper introduced the architecture and information model of the system.

The proposed session based collaboration environment is expected to efficiently and effectively support a BIM-based collaboration process in the AEC industry.

\section{ACKNOWLEDGEMENT}

This work was supported by the Korean Institute of Construction \& Transportation Technology Evaluation and Planning (KICTEP) with the program number of "06-Unified and Advanced Construction Technology Program-E01".

\section{REFERENCES}

[1] Ronen Barak, Yeon-Suk Jeong, Rafael Sacks, Charles Eastman (2007), "Unique Requirements of Building Information Modeling for Cast-in-Place Reinforced Concrete", Computing in Civil Engineering.

[2] H. Kim, F. Grobler (2007), "Building Ontology to Support Reasoning in Early Design", Computing in Civil Engineering.

[3] Lee, G. Sacks, R. and Eastman, C. M. (2006). "Specifying parametric building object behavior (BOB) for a building information modeling system". Automation in Construction, 15(6), 758-776.

[4] Chimay J. Anumba, John M. Kamara and AnneFrancoise Cutting-Decelle, Concurrent Engineering in Construction Projects, Taylor \& Francis 2007.

[5] Webster (2008), www.webster.com, Last accessed on Jan. 10, 2008. 